\title{
Efficacy and Costs of Two Forms of Stress Management Training for Cancer Patients Undergoing Chemotherapy
}

\author{
By Paul B. Jacobsen, Cathy D. Meade, Kevin D. Stein, Thomas N. Chirikos, Brent J. Small, and John C. Ruckdeschel
}

\begin{abstract}
Purpose: Professionally administered psychosocial interventions have been shown to improve the quality of life of cancer patients undergoing chemotherapy. The present study sought to improve access to psychosocial interventions during chemotherapy treatment by evaluating the efficacy and costs of a patient self-administered form of stress management training that requires limited professional time or experience to deliver.

Patients and Methods: Four hundred eleven patients about to start chemotherapy were randomly assigned to receive usual psychosocial care only, a professionally administered form of stress management training, or a patient self-administered form of stress management training. Quality-of-life assessments were conducted before randomization and before the second, third, and fourth treatment cycles. Intervention costs were estimated from both payer and societal perspectives.

Results: Compared with patients who received usual care only, patients receiving the self-administered in-
\end{abstract}

$\mathrm{T}$ HE ADVERSE EFFECTS of chemotherapy on quality of life are well documented. In addition to such common side effects as nausea ${ }^{1}$ and fatigue, ${ }^{2}$ many patients experience heightened emotional distress ${ }^{3,4}$ and interference with physical, social, and role functioning ${ }^{5}$ as a consequence of receiving chemotherapy. Although much of the effort to improve quality of life has focused on the evaluation of pharmacologic agents, such as antiemetic medications, ${ }^{6}$ a number of randomized clinical trials have documented the benefits of psychosocial interventions for patients undergoing chemotherapy treatment. ${ }^{7,8}$

Early studies in this area focused on the use of psychosocial interventions to improve quality of life among chemotherapy patients who had developed anticipatory nausea and vomiting. This research demonstrated that a variety of professionally administered interventions, including progressive muscle relaxation training with guided imagery, ${ }^{9,10}$ systematic desensitization, ${ }^{11}$ and biofeedback, ${ }^{12}$ were effective in relieving nausea, vomiting, and emotional distress in the period just before chemotherapy administration. Some of the same interventions were also found to have beneficial effects on nausea, vomiting, and emotional distress in the hours and days after chemotherapy administration. ${ }^{10,11}$

These studies encouraged further research that has proceeded in two directions. One direction has involved modifying the delivery of the interventions so that they might become more accessible to chemotherapy patients experiencing aversive treatment side effects. In the studies de- tervention reported significantly $(P \leq .05)$ better physical functioning, greater vitality, fewer role limitations because of emotional problems, and better mental health. In contrast, patients who received the professionally administered intervention fared no better in terms of quality of life than patients receiving usual care only. Costs of the self-administered intervention were estimated to be $66 \%$ (from a payer perspective) to $68 \%$ (from a societal perspective) less than the average costs of professionally administered psychosocial interventions for patients starting chemotherapy.

Conclusion: Evidence regarding the efficacy and favorable costs of self-administered stress management training suggests that this intervention has the potential to greatly improve patient access to psychosocial intervention during chemotherapy treatment.

J Clin Oncol 20:2851-2862. ๑ 2002 by American Society of Clinical Oncology.

scribed above, the interventions were typically delivered by a mental health professional on an individual basis. Widespread availability of interventions delivered in this manner is unlikely due to the limited number of qualified mental health professionals working in oncology settings as well as the potentially prohibitive costs of using mental health professionals as interventionists. In one effort to enhance access, Morrow et al ${ }^{13}$ developed a behavioral treatment for chemotherapy-induced nausea and vomiting that could be delivered by oncologists and oncology nurses and compared its efficacy to the same treatment delivered by psychologists and a no intervention control condition. Administration of the behavioral treatment (systematic desensitization) required patients to be seen on an individual basis for two 1-hour sessions. Delivery of the intervention by oncology professionals was found to be as effective as delivery by

From the H. Lee Moffitt Cancer Center and Research Institute and University of South Florida, Tampa, FL.

August 21, 2001; accepted March 19, 2002.

Supported by grant no. R01 CA70875 from the National Cancer Institute, Bethesda, MD, and grant no. PBR-99 from the American Cancer Society, Atlanta, GA.

Address reprint requests to Paul B. Jacobsen, PhD, H. Lee Moffitt Cancer Center, 12902 Magnolia Dr, MOD3-PSY, Tampa, FL 33612; email: jacobsen@moffitt.usf.edu.

(C) 2002 by American Society of Clinical Oncology.

0732-183X/02/2012-2851/\$20.00 
psychologists and superior to no intervention in relieving anticipatory nausea, anticipatory vomiting, and posttreatment nausea. Despite these positive findings, widespread adoption of this approach is unlikely due to the amount of professional time still required to deliver the intervention. In another effort to improve access, Carey and Burish ${ }^{14}$ developed progressive muscle relaxation training and guided imagery interventions that could be delivered either by paraprofessional volunteers or prerecorded audiotapes and compared the efficacy of each to professionally administered training and a no training control conditions. All forms of training were delivered during three sessions of approximately 85 minutes total duration. Consistent with previous research, patients trained by professional therapists reported less anxiety during chemotherapy and greater food intake in the 3 days after chemotherapy than patients in the no training condition. Comparisons of patients receiving paraprofessional training or audiotaped training with patients receiving no training generally yielded nonsignificant results, suggesting that these alternatives to professional training were ineffective in improving quality of life.

The other research direction has been to explore the benefits of delivering a psychosocial intervention before chemotherapy starts. Rather than wait for aversive side effects to develop, psychosocial interventions might be used to prepare patients for the expected challenges to quality of life. In the first study to examine this approach, Burish et $\mathrm{al}^{15}$ randomized patients about to start chemotherapy to a behavioral intervention condition or a no intervention control condition. The intervention consisted of one to three sessions of professionally administered training in progressive muscle relaxation and guided imagery before the start of chemotherapy, plus additional contacts with the trainer before each of the first five infusions. Compared to the control condition, patients who received training reported less anticipatory nausea, less posttreatment nausea and vomiting, and less anticipatory and posttreatment emotional distress. Two other studies have yielded similar results. Lerman et $\mathrm{al}^{16}$ found that patients who received professionally administered relaxation training and a brief orientation to treatment before the start of chemotherapy experienced less posttreatment nausea than patients randomized to a no training control condition. Likewise, Walker et $\mathrm{al}^{17}$ found that patients who received training in relaxation and guided imagery before the start of chemotherapy reported less psychological distress and better overall quality of life than patients in a standard treatment control condition. In a more comprehensive approach, Burish et $\mathrm{al}^{18}$ evaluated the benefits of a coping preparation package for patients about to start chemotherapy. This 90-minute intervention included a tour of the oncology clinic, provision of videotaped and written materials about coping with chemotherapy, and a discussion session with a therapist. Using a randomized trial design, the efficacy of this intervention was compared with relaxation training only before the start of chemotherapy, a combined coping preparation and relaxation training intervention, and a standard treatment control condition. Patients who received coping preparation reported less anticipatory nausea, less depression, and less interference in daily life from their disease and its treatment than patients who did not receive coping preparation. Taken together, these studies suggest that routinely providing psychosocial interventions to cancer patients before the start of chemotherapy may be effective in preserving quality of life over the course of treatment.

The present study sought to further these two lines of research by developing and evaluating two forms of stress management training for cancer patients about to start chemotherapy. Each form of stress management training was designed to provide instruction in three techniques (abdominal breathing, progressive muscle relaxation training with guided imagery, and coping self statements) found previously to be effective in improving physical and emotional well-being in a variety of clinical populations. ${ }^{19-21}$ In the professionally administered stress management training (PSMT) form, training was provided during a single 60minute session conducted by a mental health professional. In the patient self-administered stress management training (SSMT) form, training was provided via a package of instructional resources distributed by a mental health professional. Both forms of stress management training were expected to result in improved quality of life over the course of chemotherapy relative to a usual care only (UCO) control condition; however, the SSMT intervention was expected to be a more economically efficient approach. Toward this end, the present study also compared the costs of the two stress management interventions with each other and with other empirically studied psychosocial interventions for chemotherapy patients.

\section{PATIENTS AND METHODS}

\section{Participants}

To be eligible, participants had to (1) be diagnosed with cancer, (2) not have received intravenous chemotherapy previously, (3) be scheduled to receive a minimum of four cycles of intravenous chemotherapy with a minimum of 7 days between cycles, (4) not be scheduled to receive radiation therapy before the start of the fourth chemotherapy cycle, (5) be capable of speaking and reading English, and (6) be able to give written informed consent before study entry.

Of 507 patients approached, $96(19 \%)$ declined to participate. The remaining 411 patients provided informed consent and were randomized to one of the three intervention conditions: $\mathrm{UCO}(\mathrm{n}=137)$, PSMT $(\mathrm{n}=134)$, or SSMT $(\mathrm{n}=140)$. Twenty-nine participants withdrew or 
became ineligible before receiving the intervention to which they were assigned or completing the first follow-up quality-of-life assessment (UCO, $\mathrm{n}=5$; PSMT, $\mathrm{n}=9$; SSMT, $\mathrm{n}=15$ ). Reasons for becoming ineligible included mortality as well as changes in treatment plans resulting in chemotherapy not being administered, being administered at another institution, or being administered concurrently with radiation therapy. The remaining 382 participants all completed at least one of the follow-up assessments and were included in the quality-of-life outcome analyses described below.

These 382 participants ranged in age from 26 to 88 years old (mean, 56 years; SD, 12 years). The majority of the participants was female (76\%), white (90\%), currently married (71\%), and had attended college $(63 \%)$. Median household income was in the $\$ 40,000$ to $\$ 59,999$ range. Performance status, as measured by the Eastern Cooperative Oncology Group scale, ${ }^{22}$ was characterized as: fully active $(55 \%)$, restricted in physically strenuous activity (39\%), ambulatory but unable to carry out work activities (5\%), or capable of carrying out only limited self-care (1\%). Although a range of cancer diagnoses was sampled, $58 \%$ of participants underwent chemotherapy for treatment of breast cancer. Additional common diagnoses were lung cancer $(21 \%)$, ovarian cancer $(5 \%)$, lymphoma (4\%), colon cancer $(3 \%)$, prostate cancer $(2 \%)$, and endometrial cancer $(2 \%)$. Fourteen percent of participants reported any previous use of stress management techniques (ie, relaxation methods or abdominal breathing).

The equivalence of the three conditions on demographic and medical variables was assessed using $\chi^{2}$ tests for categorical variables and analysis of variance for continuous variables. Analyses based on the sample of 382 patients revealed no significant $(P \leq .05)$ differences between the three treatment conditions on any of the demographic or medical variables described previously.

Ninety-one of the 382 patients did not complete at least one of the three follow-up quality-of-life assessments (UCO, $\mathrm{n}=33$; PSMT, $\mathrm{n}=$ 23; SSMT, $\mathrm{n}=35$ ). This number includes 16 patients who chose to withdraw from the study, 21 patients who could not be assessed at the scheduled time, and 54 patients who became ineligible for reasons similar to those described above.

\section{Procedure}

Participants were recruited at the Moffitt Cancer Center after consultation with a medical oncologist where plans for chemotherapy administration were discussed and agreed on. After provision of written informed consent, a baseline (time 1 [t1]) assessment was conducted in a clinic waiting area or consultation room, and participants were randomized in equal numbers to one of the three intervention conditions. Randomization was conducted using an automated telephonebased system. After registering a participant through touch-tone data entry, the computer generated intervention assignment was communicated to the research assistant via synthesized speech. Participants randomized to either of the stress management interventions subsequently met with a clinician during this visit or another outpatient visit that occurred before the start of chemotherapy. Follow-up assessments were conducted with all participants in the chemotherapy clinic just before the start of the second chemotherapy cycle (time 2 [t2]), the third chemotherapy cycle (time 3 [t3]), and the fourth chemotherapy cycle (time $4[\mathrm{t} 4]$ ). In addition, participants in the PSMT and SSMT conditions only were asked just before their first infusion to complete additional items assessing intervention credibility. Using seven-point scales, these individuals rated the anticipated effectiveness of stress management training in coping with chemotherapy (1, not at all effective to 7, extremely effective) and the perceived importance of making stress management training available to other chemotherapy patients (1, not at all important to 7, extremely important). Practice diaries, which had been distributed to these participants along with intervention materials, were also collected at the first infusion. Participants recorded in these diaries the number of times each day they practiced each of three intervention components (ie, deep breathing, progressive muscle relaxation, and coping self-statements). Use of the intervention components after the start of chemotherapy was assessed by having the participants complete similar forms at $\mathrm{t} 2$ to $\mathrm{t} 4$.

Psychosocial UCO. For purposes of this study, all participants received a standardized form of psychosocial care representative of the care typically provided at the study site to patients undergoing chemotherapy treatment. In terms of procedures, all participants underwent a brief clinical evaluation performed by an oncology social worker either in person or over the telephone. The evaluation was conducted either before the start of treatment or early in the course of treatment. As part of this evaluation, the social worker assessed the patient's need for services, evaluated the patient's understanding of the medical situation, screened for the possible presence of a mood or substance use disorder, and provided information about resources available at the cancer center and in the community (eg, support groups and transportation services). The social worker informed the primary treatment team of patients who lacked an adequate understanding of their condition and arranged for services as required. Patients who evidenced mood or substance use problems were routinely referred for in-house psychiatric consultations. Adherence to the psychosocial UCO protocol was monitored by having the social worker meet regularly with the investigative team to review patient contacts and discuss the care being provided.

PSMT. In addition to receiving psychosocial UCO, participants randomized to the PSMT condition met individually with a clinician in a private clinic area for approximately 60 minutes before the initiation of chemotherapy treatment. The session began with a review of the common sources and manifestations of stress in chemotherapy patients and a discussion of the use of stress management techniques to improve mental and physical well-being. Participants then received training in the use of paced abdominal breathing as a method of relaxation. ${ }^{23}$ This instruction was followed by an active relaxation exercise that was similar to one developed by Burish et al. ${ }^{14,18}$ The exercise combined abbreviated progressive muscle relaxation training with use of relaxing mental imagery. Patients were directed to repeatedly tense and relax a standard set of muscle groups, after which they were assisted in visualizing a tranquil nature scene to enhance and sustain feelings of relaxation. Finally, participants received brief instruction in the use of coping self-statements using techniques borrowed from stress inoculation training. ${ }^{24}$ Specifically, they were instructed to stop when they sensed they were experiencing symptoms of stress, think about negative things they might be saying to themselves about their ability to cope, and focus instead on more positive thoughts about coping with stress. As part of this exercise, participants were encouraged to identify specific coping self-statements they might use during chemotherapy treatment. At the conclusion of the session, the clinician prescribed daily practice of the three techniques before the start of chemotherapy and provided participants with an audiotaped recording of the session for practice purposes. Participants were also provided with specific recommendations for use of the three techniques once chemotherapy started. The clinician subsequently met with the patient for approximately 5 minutes in the chemotherapy clinic just before the first treatment cycle to answer any questions and encourage the use of the three techniques after the start of chemotherapy. Trained doctoral-level psychologists served as clinicians. Audiotaped recordings of training sessions were reviewed periodically to monitor adherence to the standard protocol for delivery of the PSMT. 
SSMT. In addition to receiving psychosocial UCO, participants randomized to the SSMT condition met individually with a clinician for approximately 10 minutes before the initiation of chemotherapy treatment. During this meeting, the clinician provided the participant with the packet of instructional resources to be used for SSMT and explained their use. The packet included a 15-minute prerecorded videotape and an accompanying 12-page booklet developed specifically for the study that were both entitled Coping With Chemotherapy. In addition, participants received a 35-minute prerecorded audiotape entitled Active Relaxation (Leo Media, Inc, Urbana, IL). Participants were instructed to first view the videotape and then follow the directions in the booklet regarding further training, practice, and use of stress management techniques. Every effort was made to duplicate in these materials the content of the PSMT intervention. The videotape and booklet covered the same points about sources and manifestations of stress during chemotherapy and potential benefits of stress management training included in the overview portion of the PSMT intervention. The videotape and booklet also included brief instructions in paced breathing, progressive muscle relaxation with guided imagery, and use of coping self-statements similar to that covered in the PSMT intervention. Recommendations in the videotape and booklet for practicing the techniques before the start of chemotherapy and using them after the start of treatment mirrored those provided in the PSMT intervention. Interspersed throughout the videotape and booklet were testimonials provided by former chemotherapy patients about the benefits for mental and physical well-being by using the three stress management techniques. The audiotape provided to participants contained the same training exercise in progressive muscle relaxation with guided imagery that was used in the PSMT intervention. The clinician who provided the materials subsequently met with the patient in the chemotherapy clinic for approximately 5 minutes just before the first treatment cycle to answer any questions that may have come up and encourage the use of the three techniques after the start of chemotherapy. The same doctoral-level psychologists who conducted the PSMT interventions served as clinicians. Audiotaped recordings of instructional sessions were reviewed periodically to monitor adherence to the standard protocol for delivery of the SSMT.

\section{Measures}

Demographic and personal data were obtained at baseline through the use of a standard self-report questionnaire. Variables assessed included age, sex, race/ethnicity, marital status, annual income, educational level, and previous use of stress management techniques. Medical information was obtained through review of patients' medical charts. Variables assessed in this fashion included cancer diagnosis, performance status, ${ }^{22}$ and dates of chemotherapy treatment.

The Medical Outcomes Study 36-Item Short Form (SF-36) served as the primary outcome measure. ${ }^{25,26}$ This self-report instrument contains eight multi-item scales: general health perceptions, physical functioning, role limitations due to physical problems, bodily pain, general mental health, vitality, role limitations due to emotional problems, and social functioning. The SF-36 also yields two summary scores that reflect the two-dimensional factor structure underlying the eight subscales, which are a physical component summary score and a mental component summary score. Alpha coefficients for SF-36 scales at baseline in the present study ranged from 0.72 to 0.92 . The SF-36 was administered at $\mathrm{t} 1$ to $\mathrm{t} 4$.

In light of the frequent occurrence of nausea among patients receiving chemotherapy, two questions about nausea were added to the SF-36. Using the same format as the SF-36 bodily pain scale, patients were asked to rate for the past week the severity of nausea (1, none to
6 , very severe) and the degree to which nausea interfered with normal work $(1$, not at all to 5 , extremely). The alpha coefficient for this scale at baseline was 0.92 . The nausea scale was administered at t 1 to $t 4$.

Participants also completed the Center for Epidemiologic Studies Depression Scale (CES-D) and the State-Trait Anxiety Inventory A-State Scale (STAI-S) to provide additional information about the mental component of quality of life. The CES- $\mathrm{D}^{27}$ is a 20 -item questionnaire that yields a total score reflecting the severity of depressive symptomatology in the past week. The STAI-S ${ }^{28}$ is a 20 -item questionnaire that yields a total score reflecting current (ie, state) anxiety. Both the CES-D and the STAI-S were found to have adequate internal consistency at baseline in the current study (alpha $=$ 0.89 and 0.94 , respectively). These measures were administered at t1 to t4.

\section{Data Analysis}

The quality-of-life outcome analyses were designed to test the hypothesis that participants who received either form of stress management training (PSMT or SSMT) plus usual psychosocial care would experience better quality of life during chemotherapy treatment than patients receiving UCO. In the absence of a compelling theoretical or empirical rationale, no hypothesis was offered regarding the relative efficacy of the two forms of stress management training. Accordingly, the planned analyses focused on comparing each form of stress management training separately with UCO. As described previously, self-report quality-of-life indicators were administered at $\mathrm{t} 1$ and on $\mathrm{t} 2$, $\mathrm{t} 3$, and $\mathrm{t} 4$. To maximize the use of available data, analyses were performed using SAS PROC MIXED ${ }^{29}$ (SAS Institute, Cary, NC) based on an unstructured covariance matrix model assumption. The specific effects that were modeled included the main effects of group and time, the interaction between group and time, the quadratic time effect, and the quadratic interaction between group and time. Quadratic terms were entered to determine whether there might be evidence of nonlinear intervention effects (eg, plateaus or decrements in quality of life after improvement). Of particular interest was the significance of the group-by-time interactions, which would indicate a differential improvement in quality of life as a function of intervention condition. If a significant $(P \leq .05)$ interaction between group and time was observed, additional analyses were conducted to confirm that improvements in quality of life occurred among participants randomized to the stress management training condition. Similar analyses were performed to explore differences in quality-of-life outcome between participants randomized to the two forms of stress management training.

Economic aspects of the stress management interventions were evaluated by means of simple cost comparisons. We priced the value of all resources used in conducting each of our interventions as well as a set of other psychosocial interventions designed to achieve similar outcomes. ${ }^{14-18}$ Costs were computed from the two common perspectives of payer and society. ${ }^{30}$ The first refers to the value of those human and capital resources required to carry out the intervention for which reimbursement might be sought from third-party payers; the second refers to the value of all human and capital resources used in the intervention, regardless of whether reimbursement might be sought or payment actually made. The primary difference between these two perspectives relates to whether costs encompass the value of the patient's time. Because the interventions include potential substitutions between professional and patient time inputs, the societal perspective mandates the inclusion of these patient-related opportunity costs. Costs were measured by first detailing the type and amount of each resource input required to carry out each of the interventions included in the analysis. In every case, the published description of the intervention was examined to determine the amount of time required for each type 
Table 1. Cost Parameters*

\begin{tabular}{|c|c|c|}
\hline Resource Input & Unit & $\begin{array}{l}\text { Unit Cost } \\
\text { (\$) }\end{array}$ \\
\hline \multicolumn{3}{|l|}{ Human resources } \\
\hline Clinician/PhD-level therapist $†$ & One hour & 69.93 \\
\hline Baccalaureate-level trainerf & One hour & 17.50 \\
\hline \multicolumn{3}{|l|}{ Patient opportunity cost } \\
\hline Lower bound & One hour & 5.15 \\
\hline Upper bound & One hour & 15.80 \\
\hline \multicolumn{3}{|l|}{ Overhead and related } \\
\hline Clinician/PhD-level therapist† & $34 \%$ Clinician hour & 23.80 \\
\hline Baccalaureate-level trainerf & $22 \%$ Trainer hour & 3.85 \\
\hline \multicolumn{3}{|l|}{ Home } \\
\hline Lower bound & $22 \%$ Patient hour & 1.13 \\
\hline Upper bound & $22 \%$ Patient hour & 3.48 \\
\hline \multicolumn{3}{|l|}{ Intervention-specific supplies§ } \\
\hline \multicolumn{3}{|l|}{ Audiocassette } \\
\hline Commercial product & One copy & 12.50 \\
\hline Local product & One copy & 1.50 \\
\hline Brochures and related & One set & 1.78 \\
\hline Videocassettes & One copy & 2.20 \\
\hline Blank audiotapes & One & 1.20 \\
\hline
\end{tabular}

*Dollar values assigned to respective human (time) or capital inputs used in producing behavioral interventions, as described in the text.

†Medicare Fee Schedule Relative Value Units (RVUs) for CPT code 90806 , a 45-50 minute individual psychotherapy session delivered by a professional clinical psychologist, and a conversion factor of $\$ 36.6137$ are used to approximate the hourly market value of a clinician. Note that fully implemented facility RVUs for calendar year 2000 work and malpractice expense RVUs $(1.86+0.05)$ value an hour of clinician time per se, whereas the value of the associated overhead is approximated by practice expense relative value units (0.65). Source: Health Care Financing Administration (http://www.hcfa.gov/ stats/cpt/rvudown.htm).

₹Authors' estimate based on mean hourly earnings of Medical Social Workers (Source: United States Bureau of Labor Statistics).

$\S$ Authors' estimate based on prevailing market prices in Tampa, $\mathrm{FL}$, during 1999-2000.

of interventionist, the number and type of supplies and training aides, and the amount of time required for each patient to be trained in and practice the intervention. Prevailing market values circa 1999 to 2000 were then used to assign dollar amounts to the inputs and thereby calculate the cost of each intervention (Table 1). Because of its central importance to the analysis, we used the Medicare Fee Schedule to value the time of professional psychotherapists, multiplying the number of hours per minutes of professional therapist time in each intervention by this value, and then adding the respective overhead cost. We bracketed the value of patients' opportunity time costs by imputing the national hourly minimum wage as a lower bound and the prevailing mean hourly earnings of all United States workers in 2000 as an upper bound. Given the absence of sufficiently detailed data to estimate or impute opportunity costs of time directly, this range of figures provides a reasonable approximation of the economic worth of patients' time for analytic purposes. To simplify the exposition, we report the midpoint between the lower and upper bound estimates of costs from a societal perspective.

Because the interventions considered here were carried out for relatively short periods of time, we neither adjusted costs for inflationary trends nor computed discounted present values. However, because interventions differ slightly in patient attrition, costs are computed for nominal cohorts of patients in each intervention, prorating the overall mean cost for patients who began but did not complete the requisite training. In all cases, estimated costs net out any expenses of conducting the research per se, and they are taken to be incremental differences between the interventions in question and UCO delivered to cancer chemotherapy patients. By assumption, the costs of UCO are identical across all comparisons in this analysis.

\section{RESULTS}

\section{Attrition and Missing Data}

Preliminary analyses were performed comparing the 291 patients who provided complete data with the 91 participants who did not complete at least one of the three follow-up quality-of-life assessments. Failure to complete all the follow-up assessments was unrelated to treatment assignment $\left(\chi^{2}=2.03 ; P=.36\right)$ but was related to baseline quality of life as well as demographic and medical characteristics. Relative to patients with complete data, patients with incomplete data scored significantly $(P \leq .05)$ lower on the physical component summary $(t=2.06)$, vitality $(t=$ 3.06), social functioning ( $t=2.20)$, and physical functioning $(t=2.35)$ scales of the SF-36, as well as the supplementary nausea scale $(t=2.70)$. In addition, patients with incomplete data were significantly $(P \leq .05)$ more likely to be older $(t=$ 3.85), male $\left(\chi^{2}=15.84\right)$, have a poorer Eastern Cooperative Oncology Group performance status $\left(\chi^{2}=9.00\right)$, and have lung cancer $\left(\chi^{2}=21.62\right)$. These four demographic and medical characteristics were also significantly $(P \leq .05)$ related to lower scores on the SF-36 physical component summary scale in the current sample ( $r=.11$ to .42$)$.

\section{Intervention Credibility and Utilization}

Analyses of variance were conducted to compare the two stress management conditions with regard to intervention credibility and practice of stress management techniques before the start of chemotherapy. Intervention credibility was evaluated by comparing ratings provided by participants after receiving the stress management intervention but before receiving the first infusion of chemotherapy. These comparisons yielded no significant group differences in patients' ratings of the anticipated effectiveness of stress management training in helping them cope with chemotherapy $(\mathrm{F}=1.77, P=.18)$ or the perceived importance of making stress management training available to other chemotherapy patients $(\mathrm{F}=0.69, P=.41)$. Ratings in each group generally reflected favorable opinions about stress management training, with mean scores (range, 5.71 to 6.48) exceeding the midpoint (4) on seven-point (1 to 7) rating scales.

Practice of stress management techniques was evaluated by comparing diaries that were distributed to participants 
with intervention materials and collected before the first treatment cycle. The average length of this interval in days was similar ( $\mathrm{F}=0.92, P=.34$ ) for participants in SSMT condition (mean, 8.43; SD, 7.55) and the PSMT condition (mean, 9.45, SD, 7.98). An initial comparison indicated that, relative to participants in the SSMT condition, participants in the PSMT condition practiced the stress management techniques more frequently $(\mathrm{F}=9.04, P=.003)$. Subsequent comparisons indicated that this difference reflected greater practice of deep breathing $(\mathrm{F}=4.15, P=$ $.04)$ and coping self-statements $(\mathrm{F}=14.97, P<.0001)$ but not active relaxation $(\mathrm{F}=2.61, P=.11)$ by participants in the PSMT condition. Although significant differences were present, the average participant in each group practiced the techniques on a relatively frequent basis before the start of chemotherapy. For example, deep breathing was practiced an average of 5.5 times by participants in the SSMT condition and seven times by participants in the PSMT condition.

Use of stress management techniques after the start of chemotherapy was evaluated by entering patient self-report data into a 2 (group, SSMT and PSMT) $\times 3$ (time, cycle 1 to 2 interval, cycle 2 to 3 interval, and cycle 3 to 4 interval) mixed model design. The absence of a significant group effect ( $\mathrm{F}=0.23, P=.63$ ) or a significant group-by-time effect $(\mathrm{F}=1.45, P=.24)$ indicates there were no differences in the use of techniques related to the type of stress management intervention. In contrast, a significant time effect was observed $(\mathrm{F}=4.59, P=.01)$. Pair-wise comparisons of adjacent means indicated that this effect reflected an increase across stress management interventions in the use of techniques from the interval between the first and second cycles to the interval between the second and third cycles $(P=.004)$ that was sustained in the interval between the third and fourth cycles $(P=.11)$. For example, deep breathing was practiced an average of 18.5 times in the cycle 1 to 2 interval, 22.8 times in the cycle 2 to 3 interval, and 21.1 times in the cycle 3 to 4 interval.

\section{Quality-of-Life Outcome Analyses}

Comparisons of PSMT with UCO yielded no significant $(P \leq .05)$ group-by-time interaction effects or quadratic group-by-time interaction effects on any of the quality-oflife measures (Table 2). These results indicate that, in comparison with UCO, the PSMT intervention did not result in any significant improvements in quality of life.

In contrast, comparisons of SSMT with UCO yielded several significant $(P \leq .05)$ group-by-time interaction effects and quadratic group-by-time interaction effects (Table 3). With regard to the SF-36, group-by-time or quadratic group-by-time interaction effects were significant for the mental component summary, physical functioning, vitality,
Table 2. Comparisons of UCO With PSMT

\begin{tabular}{|c|c|c|c|c|}
\hline & \multicolumn{4}{|c|}{ Effect } \\
\hline & \multicolumn{2}{|c|}{ Group $\times$ Time } & \multicolumn{2}{|c|}{$\begin{array}{c}\text { Time } \times \text { Time } \times \\
\text { Group }\end{array}$} \\
\hline & $\mathrm{F}$ & $P$ & $\mathrm{~F}$ & $P$ \\
\hline \multicolumn{5}{|l|}{ SF-36 scale } \\
\hline Mental summary & 0.29 & .59 & 0.76 & .38 \\
\hline Physical summary & 0.02 & .90 & 0.31 & .58 \\
\hline Physical function & 3.03 & .08 & 1.46 & .23 \\
\hline Role-physical & 0.42 & .52 & 0.42 & .52 \\
\hline Bodily pain & 0.37 & .54 & 0.30 & .59 \\
\hline General health & 0.60 & .44 & 2.82 & .09 \\
\hline Vitality & 0.80 & .37 & 1.42 & .24 \\
\hline Social function & 0.09 & .76 & 0.11 & .74 \\
\hline Role-emotional & 0.76 & .39 & 0.03 & .87 \\
\hline Mental health & 0.19 & .66 & 3.34 & .07 \\
\hline Nausea & 1.58 & .21 & 1.46 & .23 \\
\hline CES-D & 0.40 & .53 & 2.51 & .11 \\
\hline STAI-S & 0.16 & .69 & 0.11 & .74 \\
\hline
\end{tabular}

role-emotional, and mental health scales. Group-by-time and the quadratic group-by-time interaction effects were also significant for both the CES-D and the STAI-S.

To characterize the nature of these significant interactions, additional analyses were conducted to identify significant $(P \leq .05)$ linear and nonlinear changes over time within either the UCO or SSMT intervention conditions. As summarized in Table 4, significant linear and nonlinear changes over time were evident within the SSMT intervention condition for each quality-of-life measure that demonstrated significant group-by-time or quadratic group-bytime interaction effects. With one exception (STAI-S), none

Table 3. Comparisons of UCO With SSMT

\begin{tabular}{|c|c|c|c|c|}
\hline & \multicolumn{4}{|c|}{ Effect } \\
\hline & \multicolumn{2}{|c|}{ Group $\times$ Time } & \multicolumn{2}{|c|}{$\begin{array}{c}\text { Time } \times \text { Time } \times \\
\text { Group }\end{array}$} \\
\hline & $\mathrm{F}$ & $P$ & $\mathrm{~F}$ & $P$ \\
\hline \multicolumn{5}{|l|}{ SF-36 scale } \\
\hline Mental summary & 7.01 & .009 & 4.19 & .04 \\
\hline Physical summary & 1.04 & .31 & 0.27 & .60 \\
\hline Physical function & 5.20 & .02 & 2.37 & .12 \\
\hline Role-physical & 1.40 & .24 & 3.23 & .07 \\
\hline Bodily pain & 3.26 & .07 & 3.29 & .07 \\
\hline General health & 0.35 & .56 & 0.10 & .75 \\
\hline Vitality & 1.91 & .17 & 4.60 & .03 \\
\hline Social function & 2.02 & .16 & 2.54 & .11 \\
\hline Role-emotional & 6.58 & .01 & 0.37 & .54 \\
\hline Mental health & 8.01 & .005 & 4.66 & .03 \\
\hline Nausea & 0.29 & .59 & 0.11 & .42 \\
\hline CES-D & 6.26 & .01 & 7.01 & .009 \\
\hline STAI-S & 4.09 & .04 & 5.18 & .02 \\
\hline
\end{tabular}


Table 4. Analyses of Significant Interaction Effects for Comparisons of UCO With SSMT

\begin{tabular}{|c|c|c|c|c|c|c|c|c|}
\hline & \multicolumn{8}{|c|}{ Effect } \\
\hline & \multicolumn{2}{|c|}{ Linear Effect UCO } & \multicolumn{2}{|c|}{ Nonlinear Effect UCO } & \multicolumn{2}{|c|}{ Linear Effect SSMT } & \multicolumn{2}{|c|}{ Nonlinear Effect SSMT } \\
\hline & $t$ & $P$ & $t$ & $P$ & $t$ & $P$ & $t$ & $P$ \\
\hline \multicolumn{9}{|l|}{ SF-36 scale } \\
\hline Mental summary & 0.74 & .46 & -0.52 & .60 & 4.19 & $<.0001$ & -3.42 & .0007 \\
\hline Physical function & 1.09 & .27 & -1.44 & .15 & 3.80 & .0002 & -3.61 & .0004 \\
\hline Vitality & -0.18 & .86 & -0.24 & .81 & 3.12 & .002 & -3.27 & .001 \\
\hline Role-emotional & 1.23 & .22 & -1.07 & .29 & 2.76 & .006 & -1.93 & .05 \\
\hline Mental health & 1.54 & .13 & -0.96 & .34 & 5.20 & $<.0001$ & -4.03 & $<.0001$ \\
\hline CES-D & -1.86 & .06 & 1.29 & .20 & -5.99 & $<.0001$ & 5.03 & $<.0001$ \\
\hline STAI-S & -4.80 & $<.0001$ & 3.97 & $<.0001$ & -8.31 & $<.0001$ & 7.18 & $<.0001$ \\
\hline
\end{tabular}

of the corresponding linear and nonlinear changes within the UCO condition were significant.

Although no hypotheses were offered, comparisons of SSMT and PSMT intervention conditions were also performed (Table 5). Significant $(P \leq .05)$ group-by-time or quadratic group-by-time interaction effects were observed for the STAI-S and the role-physical, bodily pain, and mental health scales of the SF-36.

To characterize the nature of these significant interactions, additional analyses were conducted to identify significant $(P \leq .05)$ linear and nonlinear changes over time within either the SSMT or PSMT intervention conditions. As summarized in Table 6, significant linear and nonlinear changes over time were evident within the PSMT intervention condition for each quality-of-life measure that demonstrated significant group-by-time or quadratic group-bytime interaction effects. With one exception (role-physical), linear and nonlinear changes over time were also significant within the SSMT intervention condition.

Table 5. Comparisons of SSMT With PSMT

\begin{tabular}{llllll}
\hline & \multicolumn{3}{c}{ Effect } \\
\cline { 2 - 3 } & \multicolumn{2}{c}{ Group $\times$ Time } & & \multicolumn{2}{c}{$\begin{array}{c}\text { Time } \times \text { Time } \times \\
\text { Group }\end{array}$} \\
\cline { 2 - 3 } \cline { 5 - 6 } & $\mathrm{F}$ & $\mathrm{F}$ & $P$ \\
\hline SF-36 scale & & & & \\
Mental summary & 3.01 & .08 & & 0.78 & .38 \\
Physical summary & 1.32 & .25 & & 1.14 & .29 \\
Physical function & 0.26 & .61 & & 0.02 & .88 \\
Role-physical & 3.34 & .07 & & 4.60 & .03 \\
Bodily pain & 5.00 & .03 & 1.44 & .23 \\
General health & 0.01 & .98 & 1.56 & .21 \\
Vitality & 0.20 & .66 & 1.16 & .28 \\
Social function & 2.66 & .10 & & 1.79 & .18 \\
Role-emotional & 2.26 & .13 & 0.11 & .74 \\
Mental health & 3.78 & .05 & 0.08 & .78 \\
Nausea & 3.54 & .06 & 2.71 & .10 \\
CES-D & 3.19 & .08 & 1.19 & .28 \\
STAl-S & 4.30 & .04 & 2.74 & .10 \\
\hline
\end{tabular}

To illustrate the significant linear and nonlinear changes listed in Tables 4 and 6, mean changes from baseline to each follow-up assessment on each of the outcome measure were computed for the UCO, SSMT, and PSMT intervention conditions. With regard to the SF-36, Figs 1 and 2 indicate that SSMT produced relatively larger improvements on the mental component summary, physical functioning, roleemotional, vitality, and mental health scales than did UCO at each of the follow-up assessments. As shown in Fig 3, SSMT also produced relatively larger reductions in depression and anxiety than did UCO at each of the follow-up assessments. The magnitude of changes over time within the PSMT condition on each of these measures generally lies between those observed for the SSMT and UCO conditions. With regard to the SF-36 role-physical and bodily pain scales (Fig 2), where neither SSMT nor PSMT yielded significant interactions with UCO, different patterns emerged. On the bodily pain scale, improvements were consistently greater in the SSMT condition than in the PSMT condition. On the role-physical scale, no consistent pattern of changes over time across intervention conditions was evident.

\section{Cost Comparisons}

Using procedures described previously, estimated perpatient costs were calculated for SSMT and PSMT, as well as for other psychosocial interventions for chemotherapy patients described in the research literature (Table 7). Similar patterns were observed when costs estimates were computed from a payer perspective or a societal perspective. That is, interventions using professional therapists were generally more expensive than those administered by paraprofessionals or patients themselves. Among non-PSMT interventions, SSMT had a higher per-patient cost than the audiotaped and paraprofessional interventions. It should be noted, however, that SSMT is the only one of these interventions found to yield quality-of-life outcomes statistically superior to UCO. Compared with the average cost of a PSMT intervention, SSMT 
Table 6. Analyses of Significant Interaction Effects for Comparisons of SSMT With PSMT

\begin{tabular}{|c|c|c|c|c|c|c|c|c|}
\hline & \multicolumn{8}{|c|}{ Effect } \\
\hline & \multicolumn{2}{|c|}{ Linear Effect SSMT } & \multicolumn{2}{|c|}{ Nonlinear Effect SSMT } & \multicolumn{2}{|c|}{ Linear Effect PSMT } & \multicolumn{2}{|c|}{ Nonlinear Effect PSMT } \\
\hline & $t$ & $P$ & $t$ & $P$ & $t$ & $P$ & $t$ & $P$ \\
\hline \multicolumn{9}{|l|}{ SF-36 scale } \\
\hline Role-physical & -0.27 & .78 & 0.92 & .36 & 2.05 & .04 & -2.10 & .04 \\
\hline Bodily pain & 6.27 & $<.0001$ & -4.98 & $<.0001$ & 3.86 & .0001 & -3.17 & .002 \\
\hline Mental health & 5.53 & $<.0001$ & -4.40 & $<.0001$ & 4.45 & $<.0001$ & -3.89 & .0001 \\
\hline STAI-S & -7.87 & $<.0001$ & 6.64 & $<.0001$ & -4.93 & $<.0001$ & 4.17 & $<.0001$ \\
\hline
\end{tabular}

was $\$ 100$ less expensive from a payer perspective and $\$ 140$ less expensive from a societal perspective.

\section{DISCUSSION}

The present study yielded three major findings. First, the SSMT intervention was found to be effective in improving quality of life over the course of chemotherapy treatment. Compared with patients receiving UCO, patients who received the SSMT intervention reported better physical functioning, greater vitality, fewer role limitations because of emotional problems, and better mental health. Improvements in quality of life were evident shortly after intervention (ie, at t2) and continued to be present at the final study assessment (ie, at t4).

The overall pattern of results suggests the SSMT intervention had its greatest impact on patient's mental wellbeing. This conclusion is supported by a visual comparison of the relative magnitude of the significant intervention effects and by results for the component summary scales of the SF-36. Significant differences between SSMT intervention group and the UCO group were present for the mental component summary scale but not the physical component summary scale. The greater impact of the intervention on mental health outcomes is not surprising given its psychological focus. Moreover, this pattern is consistent with meta-analytic results showing that psychosocial interventions for cancer patients have weaker effects on functional adjustment than emotional adjustment. ${ }^{8}$ Nevertheless, the

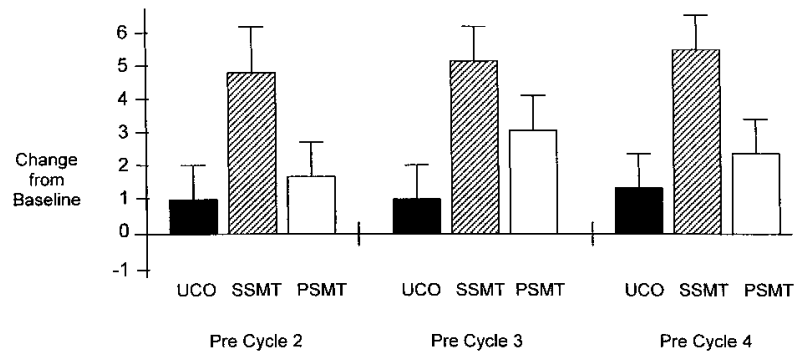

Fig 1. SF-36 mental component summary scale. findings regarding physical functioning and vitality suggest that the effects of the SSMT intervention extended beyond traditional psychosocial outcomes. Evidence of a beneficial impact on vitality is particularly noteworthy, because lack of vitality (ie, fatigue) is one of the most common and distressing symptoms experienced by cancer patients. ${ }^{31}$ The current study is the first to provide evidence that stress management training can be effective in improving energy levels in cancer patients undergoing chemotherapy.

The second major finding was that patients who received the PSMT intervention fared no better in terms of quality of life than patients who received UCO. This finding is surprising given the number of studies demonstrating beneficial effects of professionally administered relaxation training for chemotherapy patients. ${ }^{10,14-17}$ However, the current form of PSMT differed in at least two ways from relaxation training methods found previously to be effective with chemotherapy patients. First, the current intervention featured only a single training session, whereas other interventions have included as many as five training sessions. Second, other forms of training have included professionally guided relaxation sessions during the course of chemotherapy, whereas, in the current intervention, the clinician provided only guidance and encouragement during subsequent contacts. This argument fails to explain why the SSMT intervention, which did not include any professionally guided training or relaxation sessions, was more effective than UCO. The observed lack of effectiveness of the PSMT intervention cannot be attributed to problems with its credibility or with practice and use of the recommended techniques. Data collected as part of the study indicated no difference between the two forms of stress management training in patients' ratings of anticipated effectiveness. Moreover, patients randomized to the PSMT intervention practiced and used the techniques as frequently as patients randomized to the SSMT intervention. However, data are not available to rule out the possibility that patients receiving the PSMT intervention practiced for shorter periods of time and, therefore, were less skilled in the use of the techniques. By providing patients with a concise package of 

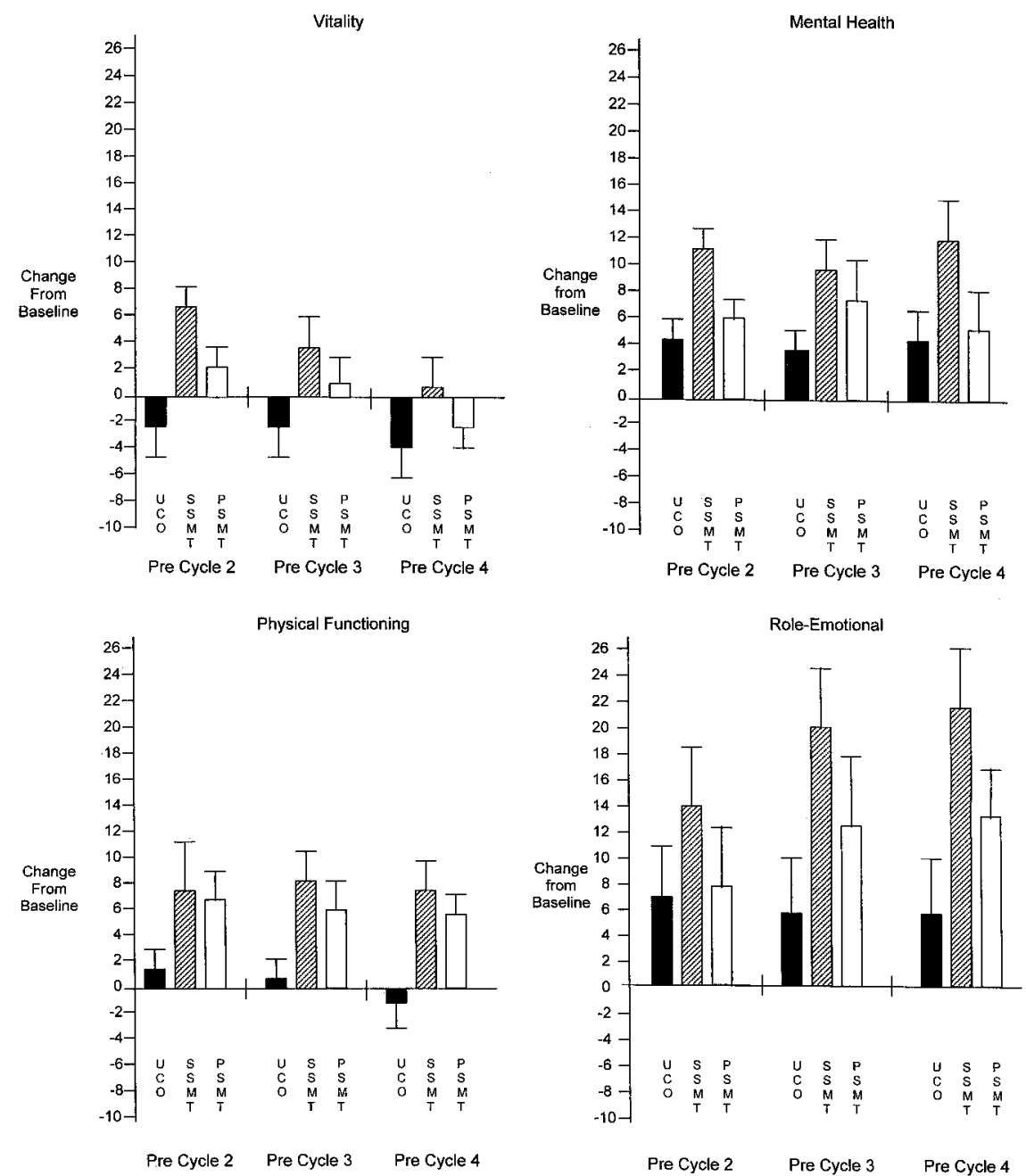

Fig 2. SF-36 scales.
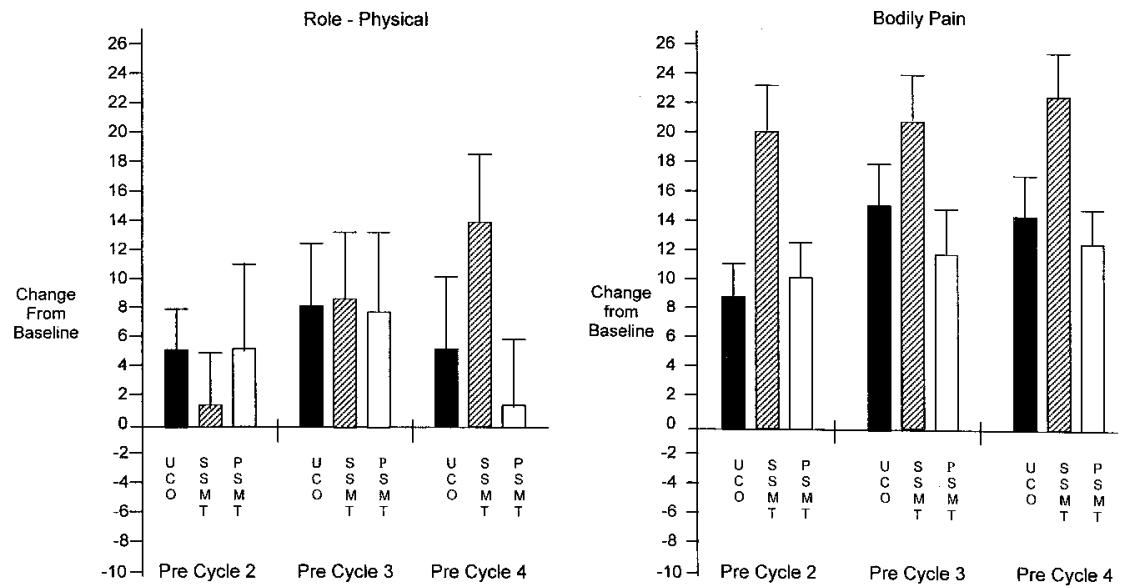

UCO = Usual Care Only

SSMT $=$ Self-Administered Stress Management Training

PSMT = Professionally Administered Stress Management Training 


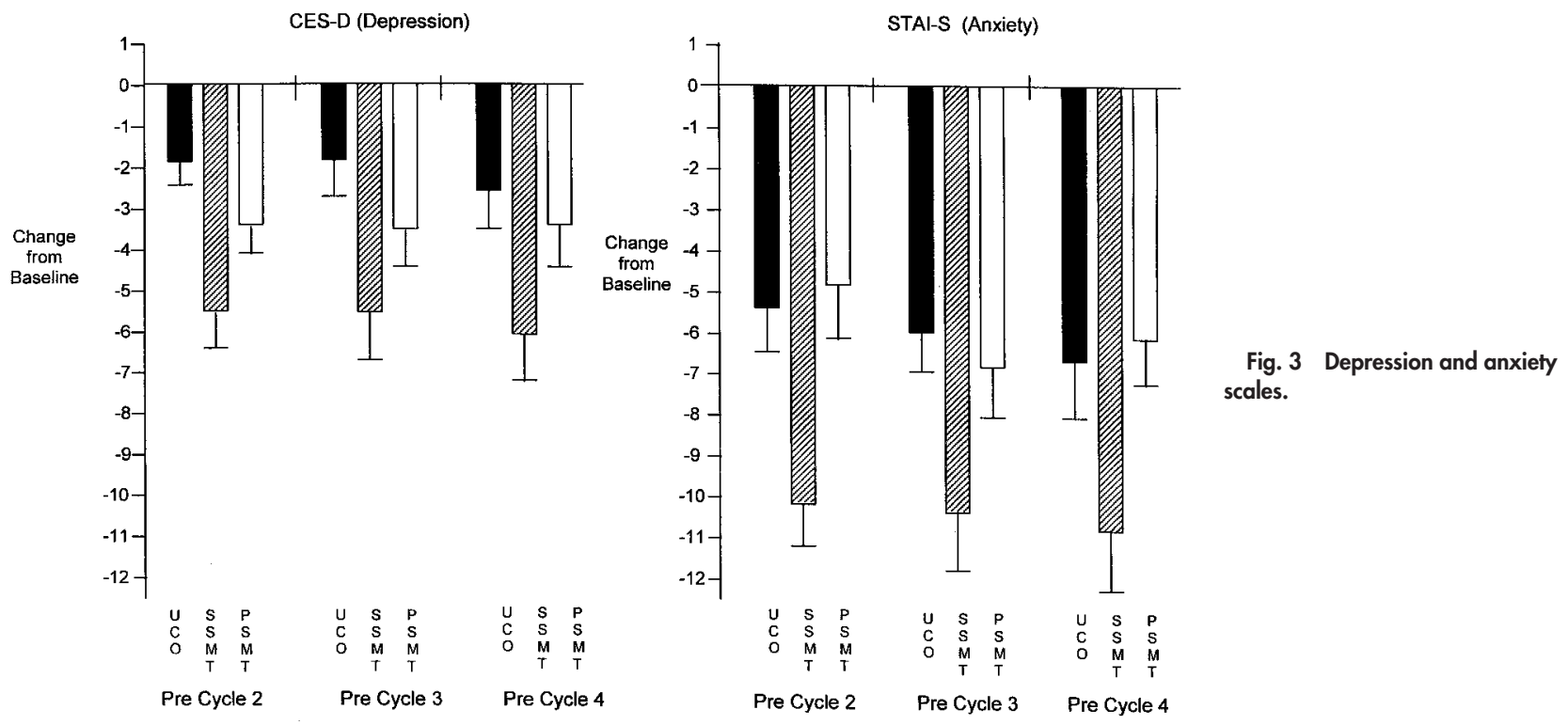

information that they could review and interact with at their own pace, the SSMT intervention may have facilitated better mastery of the stress management techniques. The use of patient testimonials about the effectiveness of stress management training exclusively in the SSMT intervention may also serve to explain the observed pattern of results. These testimonials may have encouraged greater use of the techniques in the recommended manner by patients in the

Table 7. Comparison of Incremental Costs of Psychosocial Interventions for Chemotherapy Patients

\begin{tabular}{|c|c|c|}
\hline \multirow[b]{2}{*}{ Intervention (ref) } & \multicolumn{2}{|c|}{ Costs Per Patient $(\$)^{*}$} \\
\hline & $\begin{array}{c}\text { Payer } \\
\text { Perspective }\end{array}$ & $\begin{array}{c}\text { Societal } \\
\text { Perspective }\end{array}$ \\
\hline \multicolumn{3}{|l|}{ Patient self-administered } \\
\hline Stress management training (current study) & 47 & 73 \\
\hline Relaxation training (audiotaped) $)^{14}$ & 26 & 90 \\
\hline \multicolumn{3}{|l|}{ Paraprofessionally administered } \\
\hline Relaxation training ${ }^{14}$ & 32 & 80 \\
\hline \multicolumn{3}{|l|}{ Professionally administered } \\
\hline Stress management training (current study) & 110 & 136 \\
\hline Relaxation training ${ }^{15}$ & 342 & 453 \\
\hline Relaxation training ${ }^{14}$ & 141 & 189 \\
\hline Relaxation training ${ }^{18}$ & 156 & 196 \\
\hline Relaxation training ${ }^{16}$ & 34 & 83 \\
\hline Relaxation training ${ }^{17}$ & 80 & 216 \\
\hline Coping preparation ${ }^{18}$ & 79 & 119 \\
\hline Relaxation training and coping preparation ${ }^{18}$ & 235 & 315 \\
\hline
\end{tabular}

${ }^{*}$ Mean costs adjusted for follow-up attrition and rounded to the nearest (year 2000) dollar. Societal perspective figures are the midpoint between the upper and lower bound estimates. See Table 1 for parameter values used in computing costs.
SSMT condition through a process of modeling. ${ }^{32}$ Testimonials about the effectiveness of stress management may have also resulted in more positive expectations about chemotherapy-related side effects among patients in the SSMT condition. Consistent with findings indicating that expectations about chemotherapy side effects (eg, nausea) predict subsequent symptom reports, ${ }^{33,34}$ one can suppose that patients with more positive expectations would experience better quality of life during the course of treatment. These possible explanations for the observed pattern of results should be evaluated in future research.

The third major finding was that the estimated costs of the SSMT intervention were generally less than those of PSMT psychosocial interventions for patients starting chemotherapy. The SSMT intervention cost an average of \$100 less from a payer perspective and $\$ 140$ less from a societal perspective than PSMT interventions. Implicit in these cost comparisons is the assumption that the SSMT interventions and the PSMT interventions are of equivalent effectiveness. The use of different outcomes measures across studies rules out the possibility of conducting direct comparisons of the relative cost-effectiveness of the two types of interventions. One goal for future research would be to achieve sufficient standardization in the measurement of quality-of-life outcomes to permit such comparisons. In the interim, it is possible to perform a rough comparison of the effectiveness of the SSMT intervention with other psychosocial interventions for cancer patients. Meta-analytic findings ${ }^{8}$ indicate that the average effect size of psychosocial interventions on 
emotional adjustment in cancer patients is $d=0.24$. The estimated effect size for the SSMT intervention relative to UCO on the SF-36 mental component summary scale ( $\mathrm{d}=$ 0.20) compares favorably with this value. In other words, evidence suggests that the SSMT intervention was able to yield improvements in emotional adjustment similar to other psychosocial interventions at $66 \%$ to $68 \%$ less than the average cost of a PSMT intervention.

In addition to cost, there are two other advantages of the SSMT intervention over PSMT interventions. First, the SSMT intervention requires less time to initiate. In the current study, 10 minutes were required to instruct patients in the SSMT intervention versus 60 minutes for the PSMT intervention. Second, the SSMT intervention requires less professional training to deliver. The PSMT intervention used in the present study required several hours of clinical instruction on how to administer the three intervention components. In contrast, the SSMT intervention required less than 1 hour of clinical instruction on how to explain the intervention to patients and answer common questions. Although a psychologist initiated the SSMT intervention in the current study, it is quite feasible to have members of the primary oncology team (eg, nurses and social workers) or even paraprofessional volunteers deliver the intervention after brief instruction. This approach could further reduce the cost of the intervention and facilitate its dissemination in oncology settings. We are currently planning a clinical trial designed specifically to evaluate the effectiveness and costs of the SSMT intervention when delivered by oncology clinic staff.

Results for present study were based on data from 382 participants who completed the baseline assessment and at least one of the three follow-up assessments. Included in this group were 91 participants (24\% of the sample) who did not complete all three of the follow-up assessments. Not surprisingly, these individuals were found to have a poorer quality of life and performance status at baseline than individuals providing complete data. Because the presence of missing data was unrelated to treatment assignment, findings regarding efficacy are not confounded by the greater likelihood of sicker patients not completing all the follow-up assessments. However, because healthier participants contributed more data, caution must be used in generalizing these findings to the population of cancer patients starting chemotherapy treatment.

In summary, the present study is the first to demonstrate the effectiveness of a SSMT intervention in improving the quality of life of patients undergoing chemotherapy for cancer. Of particular significance is evidence suggesting that the intervention yields improvements in quality of life similar to those demonstrated previously for PSMT interventions but at a much more favorable cost. The development of an effective SSMT program that requires minimal professional training and time to deliver, and costs only a modest amount more than UCO, has the potential to greatly improve chemotherapy patients' access to a psychosocial intervention. By improving access, many patients who would otherwise never receive a psychosocial intervention could experience the benefits of improved quality of life during chemotherapy treatment.

\section{ACKNOWLEDGMENT}

We thank Thomas Burish and William Redd for their consultation on the design of the study and Mary Coffeen, Lora Azzarello, and Heidi Grenke for their assistance in carrying out the study.

\section{REFERENCES}

1. Roscoe JA, Morrow GR, Hickok JT, et al: Nausea and vomiting remain a significant clinical problem: Trends over time in controlling chemotherapy-induced nausea and vomiting in 1413 patients treated in community clinical practices. J Pain Symptom Manage 20:113-121, 2000

2. Jacobsen PB, Hann DH, Azzarello LM, et al: Fatigue in women receiving adjuvant chemotherapy for breast cancer: Characteristics, course, and correlates. J Pain Symptom Manage 18:233-242, 1999

3. Morasso G, Constantini M, Viterbori P, et al: Predicting mood disorders in breast cancer patients. Eur J Cancer 37:216-223, 2001

4. Dodd MJ, Dibble S, Miaskowski C, et al: A comparison of affective state and quality of life of chemotherapy patients who do and do not develop chemotherapy-induced oral mucositis. J Pain Symptom Manage 21:498-505, 2001

5. Lutgendorf SK, Anderson B, Rothrock N, et al: Quality of life and mood in women receiving extensive chemotherapy for gynecologic cancer. Cancer 89:1402-1411, 2000
6. Gralla RJ, Osoba D, Kris MG, et al: Recommendations for the use of antiemetics: Evidence-based, clinical practice guidelines. J Clin Oncol 17:2971-2994, 1999

7. Jacobsen PB, Hann DM: Cognitive-behavioral interventions for cancer patients, in Holland JH, Breitbart W, Jacobsen P, et al (eds): Psycho-Oncology. New York, NY, Oxford University Press, 1998, pp 717-729

8. Meyer TJ, Mark MM: Effects of psychosocial interventions with adult cancer patients: A meta-analysis of randomized experiments. Health Psychol 14:101-108, 1995

9. Burish TG, Lyles JN: Effectiveness of relaxation training in reducing adverse reactions to cancer chemotherapy. J Behav Med 4:65-78, 1981

10. Lyles JN, Burish TG, Krozely MG, et al: Efficacy of relaxation training and guided imagery in reducing the aversiveness of cancer chemotherapy. J Consult Clin Psychol 50:509-524, 1982

11. Morrow GR: Effect of the cognitive hierarchy in the systematic desensitization treatment of anticipatory nausea in cancer patients: A 
component comparison with relaxation only, counseling, and no treatment. Cogn Ther Res 10:421-446, 1986

12. Burish TG, Jenkins RA: Effectiveness of biofeedback and relaxation training in reducing the side effects of cancer chemotherapy. Health Psychol 11:17-23, 1992

13. Morrow GR, Asbury R, Hammon S, et al: Comparing the effectiveness of behavioral treatment for chemotherapy-induced nausea and vomiting when administered by oncologists, oncology nurses, and clinical psychologists. Health Psychol 11:250-256, 1992

14. Carey MP, Burish TG: Providing relaxation training to cancer chemotherapy patients: A comparison of three delivery techniques. J Consult Clin Psychol 55:732-737, 1987

15. Burish TG, Carey MP, Krozely MG, et al: Conditioned side effects induced by cancer chemotherapy: Prevention through behavioral treatment. J Consult Clin Psychol 55:42-48, 1987

16. Lerman C, Rimer B, Blumberg B, et al: Effects of coping style and relaxation on cancer chemotherapy side effects and emotional responses. Cancer Nurs 13:308-315, 1990

17. Walker LG, Walker MB, Ogston K, et al: Psychological, clinical, and pathological effects of relaxation training and guided imagery during primary chemotherapy. Br J Cancer 80:262-268, 1999

18. Burish TG, Snyder SL, Jenkins RA: Preparing patients for cancer chemotherapy: Effect of coping preparation and relaxation interventions. J Consult Clin Psychol 59:518-525, 1991

19. Carlson CR, Hoyle RH: Efficacy of abbreviated progressive muscle relaxation training: A quantitative review of behavioral medicine research. J Consult Clin Psychol 61:1059-1067, 1993

20. Fried R: Breathing as a clinical tool, in Mostofsky DI, Barlow DH (eds): The Management of Stress and Anxiety in Medical Disorders. Needham Heights, MA, Allyn \& Bacon, 2000, pp 100-118

21. Meichenbaum D: Stress inoculation training: A 20-year update, in Lehrer PM, Woolfolk RL (eds): Principles and Practice of Stress Management (ed 2). New York, NY, Guilford Press, 1993, pp 373-406
22. Zubrod CG, Schneiderman M, Frei E, et al: Appraisal of methods for the study of chemotherapy in man: Comparative therapeutic trials of nitrogen mustard and triethylene thiophosphoramide. J Chronic Dis 11:7-33, 1960

23. Turk DC, Meichenbaum D, Genest M: Pain and Behavioral Medicine: A Cognitive-Behavioral Perspective. New York, NY, Guilford Press, 1983

24. Meichenbaum D: Stress Inoculation Training. New York, NY, Pergamon Press, 1985

25. Ware JE: SF-36 Health Survey: Manual and Interpretation Guide. Boston, MA, The Health Institute, New England Medical Center, 1993

26. Ware JE: SF-36 Physical and Mental Health Summary Scales: A User's Manual. Boston, MA, The Health Institute, New England Medical Center, 1994

27. Radloff LS: The CES-D scale: A self-report depression scale for research in the general population. Appl Psychol Measure 1:385-401, 1977

28. Spielberger CD: Manual for the State-Trait Anxiety Inventory (Form Y). Palo Alto, CA, Consulting Psychologists Press, 1983

29. Little RC, Milliken GA, Stroup WW, et al: SAS System for Mixed Models. Cary, NC, SAS Institute, 1996

30. Gold MR, Siegel JE, Russell LB, et al (eds): Cost Effectiveness in Health and Medicine. New York, NY, Oxford University Press, 1996

31. Stone P, Richards M, Hardy J: Fatigue in patients with cancer. Eur J Cancer 34:1670-1676, 1998

32. Bandura A: Social Learning Theory. Englewood Cliffs, NJ, Prentice-Hall, 1977

33. Montgomery GH, Bovbjerg DH: Pre-infusion expectations predict post-treatment nausea during repeated adjuvant chemotherapy infusions for breast cancer. Br J Health Psychol 5:105-119, 2000

34. Roscoe JA, Hickok JT, Morrow GR: Patient expectations as predictors of chemotherapy-induced nausea. Ann Behav Med 22:121126,2000 